\title{
Competencias emocionales y síndrome de burnout en el profesorado de Educación Infantil Noemí Serrano-Díaz ${ }^{*}$, Margarida Pocinho² y Estíbaliz Aragón-Mendizábal ${ }^{1}$
}

\author{
'Universidad de Cádiz \\ 2 Universidad de Madeira
}

Resumen: El objetivo de este estudio fue evaluar las competencias a mejorar en la formación inicial y permanente, con el fin de prevenir el síndrome de burnout en el profesorado de dicha etapa educativa etapa. El burnout puede ejercer una influencia negativa en los procesos de enseñanza y aprendizaje, en el ambiente del aula y en los patrones de conducta que se transmiten a las niñas y niños en formación. Se estudian las posibles necesidades formativas del profesorado de Educación Infantil dirigidas al desarrollo de un equilibrio de los estados de ánimo del profesorado y a transmitir seguridad, tranquilidad y afecto, factores que contribuyen al progreso armónico e integral del alumnado. Han participado un total de 91 profesores de Educación Infantil. A todos ellos se les administró el Maslach Burnout Inventory (MBI-HSS) y el Trait Meta-Mood Scale (TMMS), utilizando un diseño ex post facto. Los resultados mostraron que los participantes presentaban niveles no significativos del síndrome de burnout. El profesorado Educación Infantil percibe poseer unos niveles altos de competencias emocionales en sus tres dimensiones (Claridad, Reparación y Atención). Asimismo, la percepción de autoeficacia en la dimensión emocional "reparación", surgió como factor de protección ante el burnout.

Palabras clave: Competencia emocional, Educación Infantil, Formación de profesorado, Síndrome de burnout.

Emocional competencies and burount syndrome in early childhood education teachers Abstract: The aim of this study was to evaluate the competences to be improved in initial and permanent formation, in order to prevent burnout syndrome in the teachers of this stage of education. Burnout may have a negative influence on the teaching and learning processes, classroom environment and behaviour patterns that are transmitted to children in training. The possible training needs of the Early Childhood Education teachers are studied in order to develop a balance of the teachers' moods and to transmit security, tranquility and affection, factors that contribute to the harmonious and integral progress of the students. A total of 91 teachers of Early Childhood Education participated. They were all administered the Maslach Burnout Inventory (MBI-HSS) and the Trait Meta-Mood Scale (TMMS), using an ex post facto design. The results showed that participants had non-significant levels of burnout syndrome. The Early Childhood Education teachers perceive that they possess high levels of emotional competence in their three dimensions (Clarity, Reparation and Attention). Likewise, the perception of self-efficacy in the emotional dimension "reparation" emerged as a factor of protection against burnout.

Keywords: Teacher training, Early Childhood Education, Burnout, Emotional competencies.

Parece que el estrés se encuentra en el origen del $50 \%$ de las bajas laborales en la Unión Europea (Guerrero y Rubio, 2005). Durán, Extremera, Montalbán y Rey (2005) mencionan que la literatura muestra evidencias de un

Recibido: 04/06/2017 - Aceptado: 24/11/2017 - Avance online: 14/12/2017

*Correspondencia: Noemí Serrano Díaz.

Universidad de Cádiz.

Código Postal: 11519, Puerto Real, España.

E-mail: noemi.serrano@uca.es

Serrano, N., Pocinho, M., y Aragón, E. (2018). Competencias emocionales y síndrome de Burnout en el profesorado de Educación Infantil. Journal of Psychology and Education, 13(1), 1-12, doi: hitp://dx.doi.org/10.23923/rpye2018.01.153 elevado nivel de estrés experimentado por el profesorado, el cual afecta negativamente a su salud física y mental, así como al ambiente de aprendizaje y al logro de los objetivos educativos de su alumnado. En España se publicó un informe de FETE-UGT (2014), en el que se realizaron 2500 evaluaciones de trabajadores de la enseñanza. Este estudio reveló que un 12,60 \% de los trabajadores que realizaron el test de medición del burnout, 
mostraba síntomas de padecer el síndrome y un 18,52 \% estaba en riesgo de padecerlo. Así mismo, en otros estudios de FETE-UGT (2004, 2006) realizados también en España se sugiere que estas bajas laborales tienen lugar entre los trimestres segundo y tercero del curso académico, por lo que se puede establecer una relación directa con el desgaste psíquico que sufre el docente a medida que avanza el curso escolar.

Son diferentes las razones que se pueden esgrimir para justificar el abordaje de esta temática: desde la prevención de riesgos laborales, pasando por su influencia en el gasto público, tanto en costes sanitarios como en aquellos gastos derivados de cubrir una baja laboral prolongada. Aunque las razones que nos han movido fundamentalmente a realizar este estudio son las consecuencias psicoeducativas del síndrome. Es decir, la incidencia que puede tener el síndrome de burnout en el proceso educativo en el aula, dada la potencial influencia negativa que ejerce el burnout en los procesos de enseñanza y aprendizaje que repercute en su alumnado. La política educativa se propone mejorar la enseñanza a través de la mejora de la calidad del profesorado (formación, definición del proceso de acceso a la profesión, entre otros aspectos). El burnout puede ser una amenaza para el buen desempeño de los profesionales de la educación, que afectaría negativamente a la calidad de su trabajo (Pocinho y Peresterelo, 2011). La existencia de un riesgo objetivo de sufrir el síndrome de burnout en los profesionales de la educación, daría sentido a la implementación de planes de prevención, evaluación y tratamiento apropiados.

En una sociedad sometida a un acelerado cambio social, laboral y tecnológico, así como una interdependencia más global, las personas, en la necesidad de afrontar estas nuevas realidades, necesitan percibir el control sobre los cursos de sus vidas y sienten la necesidad de creerse capaces de utilizar las competencias emocionales y profesionales necesarias para afrontar los distintos retos que se les presentan. Estas creencias de eficacia son un factor determinante en el estudio de las conductas saludables (Bandura, 2004;
Pocinho y Capelo, 2009). Dentro de la teoría socialcognitiva, la autoeficacia fue definida por Bandura (1997, p. 3) como "las creencias en las propias capacidades para organizar y ejecutar los cursos de acción requeridos para producir unos determinados logros futuros". De ahí la importancia de las creencias de eficacia en la prevención de riesgos psicosociales (como puede ser el burnout) en el desempeño laboral. Se considera que son cuatro sus principales fuentes: las experiencias de éxito y de dominio, la experiencia vicaria mediante la observación de logros y fracasos de los demás, la persuasión verbal, y los estados fisiológicos o activación emocional (Nunes y Farias 2008). De todos estos aspectos vinculados a la autoeficacia, las experiencias de éxito son consideradas como la más importante (Capelo y Pocinho, 2014). La experiencia de éxito incrementa las auto evaluaciones positivas de autoeficacia que, a su vez, influyen en su éxito futuro constituyendo un círculo que se retroalimenta.

Dada la importancia que tienen las competencias y el sentimiento de autoeficacia, tanto en el terreno emocional como profesional, en el ámbito organizacional de los trabajadores, hemos considerado la importancia de estos aspectos en las maestras y maestros de Educación Infantil (en adelante EI). El objetivo de este estudio fue evaluar cuáles son las competencias a mejorar en su formación inicial y permanente con el fin de prevenir el síndrome de burnout.

El síndrome burnoutse considera actualmente como una patología derivada del estrés laboral (Gil-Monte, 2001). Su definición, responde a un intento de conceptualizar teóricamente el constructo a partir de los factores obtenidos tras la administración de uno de sus instrumentos de medida, concretamente el Maslach Burnout Inventory (M.B.I) de Maslach y Jackson (1981). El síndrome se ha visto definido a partir de las dimensiones extraídas de la administración del $\mathrm{MBI}$, que presenta tres dimensiones: Agotamiento emocional, Despersonalización y falta de Realización Personal en el trabajo. El Agotamiento emocional se refiere a la pérdida de recursos emocionales. La persona que lo sufre siente no poder dar más de sí mismo en el ámbito emocional y afectivo. La 
Despersonalización (o Cinismo) se refiere a las actitudes negativas y cínicas que manifiesta el trabajador hacia las personas destinatarias de su trabajo. La Realización Personal, hace referencia a la tendencia a evaluar de forma negativa el propio trabajo, un concepto muy cercano al concepto de autoeficacia de Bandura (1999).

Trasladándolo al ámbito educativo Durán, Extremera y Rey (2004, p. 47) lo definen "como una respuesta de estado emocional negativo, generalmente acompañada por cambios fisiológicos potencialmente peligrosos, resultantes de aspectos del trabajo del maestro y mediados por la percepción de que las demandas del trabajo son amenazantes y por los mecanismos de afrontamiento que son activados para reducir esa amenaza". En este ámbito Arís-Redó (2009) diferencia cuatro grupos de síntomas para caracterizar el síndrome en los docentes: psicosomáticos (cefaleas, insomnios, dolores musculares y molestias gastrointestinales), conductuales (absentismo laboral y problemas relacionales), emocionales (distanciamiento afectivo de las personas que ha de atender) y defensivos (negación de emociones y actitudes cínicas ante los alumnos).

Existen numerosos estudios en referencia al burnout en etapas educativas superiores a Educación Infantil (Capelo, Pocinho y Santos 2013; Pena y Extremera, 2012) por lo que se muestra una laguna de investigación sobre la temática que aborda este estudio.

Respecto a las competencias emocionales del profesorado de El, interesa la inteligencia emocional percibida como aspecto mensurable dentro del ámbito de las emociones. La investigación hace hincapiés en la relevancia que ha alcanzado este tipo de competencias en la labor del docente y, concretamente, en el liderazgo pedagógico (Alberici y Serreri, 2005; Boyatzis, McKee y Goleman 2002; DonaldsonFeilder y Bond, 2004; Giardini y Frese, 2006; Hughes, 2005; Navío, 2005; Soares y Pocinho, 2013).

Mayer y Salovey evalúan la Inteligencia Emocional Percibida (en adelante IEP) mediante la escala Trait Meta-Mood Scale (TMMS-24) (Salovey et al. 1995). La evaluación de la IEP tiene sentido en el ámbito educativo en la medida que se puedan conocer también las estrategias utilizadas por los docentes para su afrontamiento (Mikolajczak, Nelis, Hansenne y Quoidbach, 2008). Igualmente, puntuaciones bajas en la IEP se relacionan con niveles altos de estrés (Austin, Saklofske y Mastoras, 2010) y con la identificación de los problemas como una amenaza, respondiendo de manera impulsiva o con estrategias de evitación (Christopher y Thomas, 2009). Brackett, Mayer, y Warner (2004) encontraron evidencias sobre la relación entre IE y menores síntomas depresivos, ansiedad y comportamientos no adaptativos, así como mejores conductas saludables y bienestar psicológico. Actualmente, la formación universitaria que recibe el alumnado del Grado en Educación Infantil se recoge en la Orden ECl/3854 (2007). En la citada orden se establecen como habilidades docentes las referidas a preservar la estabilidad emocional del profesorado para contribuir al progreso armónico e integral de los estudiantes, así como, la importancia de transmitirles seguridad, tranquilidad y afecto. Estas competencias, orientadas a afrontar las situaciones de estrés laboral y burnout y a la regulación y equilibrio de las emociones en el aula, que deben ser adquiridas por el alumnado universitario del Grado en El, se encuentran reflejadas en el módulo de formación básica, más concretamente, en el apartado dedicado a la Organización del espacio escolar, materiales y habilidades docentes, en el que explicita textualmente:

"Valorar la importancia que tienen la estabilidad y la regularidad en el entorno escolar, los horarios y los estados de ánimo del profesorado como factores que contribuyen al progreso armónico e integral de los estudiantes. Saber trabajar en equipo con otros profesionales de dentro y fuera del centro en la atención a cada estudiante. Atender las necesidades de los estudiantes y transmitir seguridad, tranquilidad y afecto" (Orden $\mathrm{ECl} / 3854,2007$, p. 53737).

Se comprueba, por tanto, como el cambio a los nuevos planes de estudio contempla y hace hincapié en el desarrollo de estas competencias básicas para el afrontamiento 
de estas dificultades laborales. Es un avance importante respecto a los antiguos planes de estudio aunque desde un punto de vista subjetivo se avanza con lentitud. En definitiva, se considera fundamental especificar las competencias recogidas en la orden que regula el título y que señala la importancia de las competencias emocionales a la hora de desempeñar la profesión como docente.

Teniendo en cuenta el modelo de referencia de la autorregulación de emociones descrito por Salovey (Salovey et al., 1995) se identifican cinco dimensiones básicas en las competencias emocionales: cooperación, asertividad, responsabilidad, empatía, autocontrol. Estas dimensiones se solapan con el concepto de inteligencia emocional, tal como lo define Goleman (1995), dividido en cinco dominios: autoconciencia emocional, manejo de las emociones, automotivación, empatía y habilidades sociales, que a su vez incluían la existencia de veinticinco competencias. Asimismo, la importancia del desarrollo de las competencias en el ámbito de la docencia del profesorado, se estima que en su formación pueden tener significativas repercusiones en el terreno profesional y en el ámbito organizacional de los centros educativos. Por ello, en este estudio se plantea como objetivo primordial conocer las competencias emocionales que tiene el profesorado de las primeras etapas educativas (a través del test TMMS) para prevenir el síndrome de burnout. De esta manera podremos conocer los déficits más significativos en este ámbito y desarrollar programas para determinar cómo mejorarlas, en su formación inicial y permanente. Entendemos como implicaciones del estudio que esta contribución podría prevenir el síndrome de burnout del profesorado, repercutiendo en última instancia en la calidad educativa.

Para conseguir este objetivo general, se plantea los siguientes objetivos específicos: (1) Conocer el nivel de burnout del profesorado de Educación Infantil en una muestra significativa. (2) Evaluar la percepción de una muestra de profesorado de Educación Infantil sobre su nivel de autoeficacia en relación a su competencia emocional. (3) Explorar la posible capacidad predictiva que tienen las competencias emocionales sobre el burnout.

Como hipótesis del estudio se plantean las siguientes: en primer lugar, el profesorado de Educación Infantil de la Bahía de Cádiz muestran niveles elevados de burnout. En segundo lugar, el profesorado de Educación Infantil de la Bahía de Cádiz se percibe con niveles bajos de autoeficacia en relación a sus competencias emocionales. Finalmente, existe una relación inversa entre el nivel de competencia emocional y burnout del profesorado de la Bahía de Cádiz.

\section{MÉTODO}

\section{PARTICIPANTES}

Los participantes en el estudio fueron un total de 91 maestras y maestros que ejercían su docencia en 21 centros públicos de la zona geográfica que abarca Bahía de Cádiz (Cádiz capital, Puerto Real, El Puerto de Santa María, San Fernando y Chiclana) en España. El nivel socioeconómico de los colegios era medio y todos ellos de Educación Infantil con alumnado de 3 a 6 años. Un 11\% eran hombres y $89 \%$ mujeres, con una edad media de 42.62 años, (Rango de 25-58; DT = 7.82).

Para la delimitación adecuada del marco muestral acudimos a la Administración Educativa para solicitar la información necesaria. Concretamente, se necesita conocer el número de centros de Educación Infantil públicos de la zona, así como la relación de puestos de trabajo de cada centro escolar. Se estableció como unidad de muestreo el Centro Escolar identificado por: nombre, situación geográfica, características (tamaño del Centro Educativo, localidad, nivel sociocultural). La estrategia definitiva de muestreo quedó establecida dividiendo la población en estratos geográficos, tomando dentro de cada estrato las unidades de forma intencional para conseguir la representación de todos los factores considerados "a priori" de interés (localidad, tamaño del centro, nivel sociocultural). Sobre cada centro muestreado se tomó al profesorado como subunidades muestrales, siendo la estrategia final de selección de la muestra un muestreo no aleatorio. En el estudio 
participaron voluntariamente el $100 \%$ de los maestros de Educación Infantil pertenecientes a los 21 centros educativos seleccionados.

\section{INSTRUMENTOS}

Los instrumentos utilizados fueron el Maslach Burnout Inventory (MBI-HSS, Maslach y Jackson, 1986), en su versión española desarrollada por Salanova y Grau (1999) y el Trait MetaMood Scale (TMMS), de Salovey et al. (1995), en su versión española de Fernández-Berrocal, Extremera, y Ramos (2004).

Maslach Burnout Inventory (MBI). Utilizado para la evaluación del burnout. El cuestionario consta de 22 ítems que se distribuyen en tres escalas denominadas: Realización personal en el trabajo (8 ítems), Agotamiento Emocional (9 ítems) y Despersonalización o cinismo (5 ítems). Los participantes valoraban cada ítem del cuestionario, siendo éste una escala en la que indicaban con qué frecuencia habían experimentado en el último año la situación descrita por el ítem. Se utilizaron puntuaciones de 0 a 6 (0, Nunca; 1, alguna vez al año o menos; 2, una vez al mes o menos; 3 , algunas veces al mes; 4, una vez a la semana; 5 varias veces a la semana; y 6 , diariamente). El cuestionario se administra individualmente y tiene una duración aproximada de 15 minutos. La fiabilidad del cuestionario para las diferentes escalas obtuvo un alpha de Cronbach de .84 para el Agotamiento emocional, .77 para Realización Personal, y .24 para la dimensión de Despersonalización, por lo que esta última no fue incluida en los cálculos del estudio. La fiabilidad completa del $\mathrm{MBI}$ fue de alpha de Cronbach $=.64$.

Trait Meta-Mood Scale (TMMS). Se utilizó para la evaluación de la Competencia Emocional Percibida (CEP). Es una escala que, valora el metaconocimiento que las personas tienen sobre sus capacidades emocionales y se realiza por medio de un autoinforme. Las tres dimensiones claves de la inteligencia emocional intrapersonal evaluadas en la escala son: (a) Atención a los propios sentimientos, que es el grado en el que las personas creen prestar atención a sus emociones y sentimientos (por ej. "Pienso en mi estado de ánimo constantemente"); (b) Claridad emocional, o grado en el que las personas creen percibir sus emociones (por ej. "Frecuentemente me equivoco con mis sentimientos"); y (c) Reparación de las propias emociones, o grado en el que el sujeto cree poder regular sus estados emocionales negativos y prolongar los positivos (por ej. "Aunque a veces me siento triste, suelo tener una visión optimista").

Este instrumento está compuesto por 22 ítems y que se agrupan en tres dimensiones: Atención a los propios sentimientos, Claridad emocional y Reparación de las emociones. A los participantes se les pide que evalúen el grado en el que están de acuerdo con cada uno de los ítems sobre una escala tipo Likert de 5 puntos, que varían desde muy de acuerdo (1) a muy en desacuerdo (5). El cuestionario se administra individualmente y tiene una duración aproximada de 20 minutos. Los valores de fiabilidad obtenidos mediante el alpha de Cronbach fueron de .80 para la dimensión de Atención a los propios sentimientos; .89 para claridad emocional; y .90 para reparación de las emociones. La fiabilidad completa del TMMS fue de alpha de Cronbach $=.90$.

\section{PROCEDIMIENTO}

A través de la jefatura de estudios de cada centro, se concertó una cita con los docentes que aceptaron participar voluntariamente en la investigación completando un consentimiento informado. Los cuestionarios fueron administrados por los responsables de la investigación quienes, al ser el test autoinformado, aseguraban que la evaluación se completase en las condiciones idóneas para ello. El test se aplicó de forma individual siguiendo las indicaciones del manual, respetando el ritmo del profesorado y horario de los centros. Se llevó a cabo en los meses de abril y mayo, es decir, en el tercer trimestre. El tiempo medio empleado en aplicar el test es de 30-40 minutos por participante.

Entendemos que la muestra puede no ser suficientemente representativa, por lo que los resultados no pretenden ser extrapolables, de modo que esta investigación constituye solo un primer acercamiento a la realidad a través del análisis de unas situaciones concretas. 


\section{ANÁLISIS DE DATOS}

Las características del estudio permitieron realizar un diseño ex post facto describiendo las características de las variables estudiadas. Los datos fueron volcados en una base de datos y tratados mediante el paquete estadístico SPSS versión 20. El análisis de los datos se ha realizado en tres aspectos: en primer lugar se expone una descripción de los resultados obtenidos por la totalidad de la muestra, posteriormente se realizaron las correlaciones directas entre las variables estudiadas por los dos cuestionarios de evaluación y finalmente se ha realizado un análisis de regresión lineal por pasos para conocer el valor predictivo de las variables de Inteligencia emocional sobre el síndrome de burnout.

\section{RESULTADOS}

Con respecto a los resultados obtenidos en relación al burnout, el cuestionario $\mathrm{MBI}$ que proporciona tres dimensiones: Agotamiento emocional, Despersonalización y Realización personal. Con respecto al cuestionario TMMS se contemplan las dimensiones: Claridad, Reparación y Atención. Se recogieron los valores medios de cada una de las dimensiones obtenidas por los participantes.

Los resultados obtenidos para la dimensión Agotamiento Emocional son de un punto por debajo de la media de estandarización del Test, por lo que que la muestra de estudio no se encuentra cansada emocionalmente en su trabajo, dado que esta dimensión se refiere a la disminución o pérdida de recursos emocionales o bien, describe sentimientos de estar saturado y cansado emocionalmente por el trabajo.

Respecto a la dimensión Realización Personal el resultado del estudio muestra una puntuación alta $(M=5.1 ; D T=0.79)$. Cuanto mayor es la puntuación en esta dimensión menor es la realización personal, dado que, en esta dimensión, la puntuación es inversamente proporcional al grado de burnout. Es decir, a menor puntuación en la dimensión Realización - Logro Personal más afectado está el sujeto con el síndrome que nos ocupa. En consecuencia, podemos decir que la muestra objeto de estudio no presenta índices elevados del síndrome de burnout.

En relación con las competencias emocionales, la escala TMMS contiene tres dimensiones claves de la Inteligencia Emocional: Atención emocional, Claridad de sentimientos y Reparación emocional. Como podemos observar en la Tabla 1, los resultados obtenidos se encuentran por encima de la media. Son unos resultados globales que podrían considerarse moderadamente altos de autoeficacia emocional. Concretamente, los resultados para la dimensión Claridad Emocional ( $M=3.72 ;$ DT $=0.74)$, nos muestran unos niveles relativamente altos de percepción en la comprensión de sus propios estados emocionales por parte del profesorado de Educación Infantil. Para la dimensión Reparación de las emociones se han obtenido puntuaciones igualmente elevadas $(M=3.81$; DT $=0.79)$, lo que nos indica que el profesorado de Educación Infantil evaluado tiene la percepción de estar capacitados para una correcta regulación de los estados emocionales. Esta es la dimensión que ha puntuado más alta en la escala TMMS. Respecto a las puntuaciones más bajas, aunque también por encima de la media, fue para la dimensión Atención a los propios sentimientos $(M=3.25 ; D T=0.80)$ que nos indicó que el colectivo objeto de estudio, percibe que es capaz de sentir y expresar los sentimientos de forma adecuada.

En relación al análisis de correlaciones entre las dimensiones del síndrome de burnout y las competencias emocionales del profesorado de El evaluado, la Tabla 1 recoge los resultados simplificados.

En referencia a la relación entre burnout y competencias emocionales se comprueba la existencia de correlaciones estadísticamente significativas entre las dos dimensiones de burnout que se toman como válidas (Agotamiento emocional y Realización personal) y dos dimensiones de la escala TMMS de competencias emocionales (Claridad y Reparación). Se comprueba que la dimensión Agotamiento emocional presenta una correlación negativa y estadísticamente significativa con las dimensión Claridad $(r=$ 


\begin{tabular}{|c|c|c|c|c|c|}
\hline \multicolumn{6}{|c|}{$\begin{array}{c}\text { Tabla l } \\
\text { Coeficientes de correlación de Pearson entre burnout y } \\
\text { competencia emocional }\end{array}$} \\
\hline Variable & 1 & 2 & 3 & 4 & 5 \\
\hline \multicolumn{6}{|l|}{$\begin{array}{l}\text { 1.Agotamiento } \\
\text { Emocional }\end{array}$} \\
\hline $\begin{array}{l}\text { 2. Realización } \\
\text { Personal }\end{array}$ & \multicolumn{4}{|l|}{$-.304^{* *}$} & \\
\hline 3. Claridad & $-.238^{*}$ & \multicolumn{3}{|l|}{$.284^{\star \star}$} & \\
\hline 4. Reparación & $-.240^{\star}$ & $.354^{* \star}$ & $542^{* *}$ & & \\
\hline 5. Atención & -.017 & & .183 & $.236^{*}$ & \\
\hline \multicolumn{6}{|c|}{$\begin{array}{l}\text { Correlación significante al nivel .05 (bilateral) * } \\
\text { Correlación significativa al nivel .01 (bilateral) ** }\end{array}$} \\
\hline
\end{tabular}

-.238, $p<.05)$ y Reparación $(r=-.240, p<$ .05). Así, a menores valores de Claridad y Reparación (lo que indicaría niveles menores de percepción de competencia emocional), mayores valores de Agotamiento emocional (lo que indicaría mayores valores de burnout). Igualmente, la dimensión Realización personal presenta una correlación positiva y estadísticamente significativa con las dimensiones Claridad ( $r=.284, p<.01$ ) y Reparación $(r=.354, p<$.05). Así, a mayores valores de Claridad y Reparación (lo que indicaría mayores niveles de percepción de competencia emocional), mayores valores de Realización personal. Se recuerda que la dimensión Realización personal, la única que está redactado en positivo, es inversamente proporcional a los valores de burnout, por lo que nos indica menores valores de este síndrome.

Para abordar el último objetivo se realizó un cálculo de regresión lineal por pasos (stepwise) introduciendo como variables predictoras las dimensiones de la competencia emocional percibida que habían mostrado correlaciones estadísticamente significativas con las distintas dimensiones de burnout. Como variables dependientes se utilizaron las distintas dimensiones de burnout. En la Tabla 2 se presenta un resumen de los principales datos obtenidos.

\begin{tabular}{|c|c|c|c|c|c|}
\hline \multicolumn{6}{|c|}{$\begin{array}{c}\text { Tabla } 2 \\
\text { Modelos del análisis de regresión resultante }\end{array}$} \\
\hline Modelo & $\begin{array}{c}\text { Variable / } \\
\text { Variables } \\
\text { predictoras }\end{array}$ & $R^{2}$ & $F$ & $B$ & $p$ \\
\hline Modelo 1 & $\begin{array}{l}\text { Agotamiento } \\
\text { Emocional }\end{array}$ & .06 & $\begin{array}{l}(1.87) \\
5.303\end{array}$ & & $.024^{*}$ \\
\hline Modelo 2 & $\begin{array}{l}\text { Realización } \\
\text { Personal }\end{array}$ & .13 & $\begin{array}{c}(12.45) \\
.354\end{array}$ & -0.024 & $.001^{*}$ \\
\hline & Reparación & & & 0.36 & \\
\hline$p<.05$ & & & & & \\
\hline
\end{tabular}

Con respecto a las dimensiones del burnout que hemos sometido a estudio (Agotamiento emocional y Realización personal) podemos comprobar que la dimensión Reparación (una de las tres dimensiones de IE) se consolida como variable predictora de ambas; más específicamente explica el $6 \%\left(R^{2}=.06\right)$ de la dimensión Agotamiento emocional. Respecto a su capacidad predictiva, un incremento en 1 punto en la dimensión Reparación reduciría .024 de la dimensión Agotamiento emocional. En relación a la dimensión Realización Personal, la dimensión Reparación explica un $13 \%\left(R^{2}=.13\right)$ de la misma. En cuanto a su capacidad predictiva, sería de esperar que un incremento en 1 punto en la dimensión en la dimensión Reparación produciría un incremento de .36 de la dimensión Realización personal. Los resultados muestran que aquellas personas que son capaces de regular sus estados emocionales (reparación) no sufren la pérdida de estos recursos (agotamiento emocional), ya que son capaces de gestionarlos adecuadamente. Del mismo modo, aquellas personas que son capaces de regular sus estados emocionales (reparación) no presentan tendencia a evaluar de forma negativa su propio trabajo (realización personal). 


\section{DISCUSIÓN}

Los resultados presentan un profesorado con niveles bajos del síndrome de burnout lo que aportaría evidencias a favor de que el profesorado de Educación Infantil no pertenece a una de las etapas educativas más afectadas por el burnout. Coinciden con los estudios de Anderson e Iwanicki (1984), Capelo, Pocinho y Santos (2013), Matud, García y Matud (2002), Schwab, Jackson y Schuler (1986), y Vandenberghe y Huberman (1999) en los que se ha encontrado una mayor prevalencia de síntomas de burnout entre el profesorado de Educación Secundaria que entre los de Primaria e Infantil. En este sentido se reitera nuestro posicionamiento al coincidir con Pena y Extremera (2012) cuando apuntan a necesidad de realizar estudios diferenciando los distintos niveles educativos, dado que se presentan niveles diferentes en las distintas etapas educativas respecto a la prevalencia del síndrome.

Los resultados del presente trabajo difieren de los obtenidos en investigaciones entre profesorado en España los cuales presentan niveles medios y en escasas ocasiones mediosaltos de burnout y estrés laboral (Aris-Redó, 2009; Extremera, Rey y Pena, 2010; Cordeiro et al., 2003 ). Por otra parte los resultados obtenidos son coincidentes con los de Bermúdez et al. (2004) quienes en una investigación realizada en la provincia de Málaga con profesorado de las etapas de Infantil y Primaria conjuntamente, estos presentaron, al igual que en la muestra estudiada, niveles bajos de burnout. Así mismo, coincidimos con investigaciones en las que se afirma que la inteligencia emocional es un recurso individual protector contra estrés y burnout ya que ayuda a regular estados de ánimo (Salovey, Mayer, Goldman, Turvey, Palfai, 1995), así como el afrontamiento a los conflictos que pueden surgir en el entorno laboral (Durán, Extremera y Rey, 2004; Limonero et al. 2004; Nikolaou y Tsaousis, 2002), actuando como protector sobre el estrés del profesorado (Brackett y Katulak, 2006; Extremera, Fernández-Berrocal y Durán, 2003).

Convenimos con Pena y Extremera (2012) quienes encuentran una relación significativa entre inteligencia emocional y burnout, como arrojan los resultados de la presente investigación y coincidimos con estos cuando sugieren que los docentes con altas niveles de inteligencia emocional son más resistentes a la posible frustración o a la aparición del síndrome burnout, ante los diferentes agentes estresores a los que se exponen en sus trabajos. Así el profesorado que presenta niveles moderadamente altos de inteligencia emocional es más resiliente al burnout dada su capacidad para saber manejar sus emociones en el entorno laboral y así reducir su nivel de estrés ocupacional sin llegar a sufrir el síndrome del maestro quemado o burnout.

La gran preocupación en los últimos tiempos por reducir el estrés y burnout en los trabajadores de cualquier tipo de organización hacen del todo necesarios estudios como el que hemos realizado. Para atenuar el efecto del síndrome es tan importante la prevención como los novedosos programas que se están implantando en las organizaciones últimamente sobre intervenciones positivas para fomentar la ilusión por el trabajo o engagement. Estas se tornan en actuaciones necesarias para ser abordadas desde un punto de vista organizacional a fin de conseguirorganizaciones cada vez más saludable a la vez que efectivas. Así mismo, estos programas redundarían en beneficio del alumnado de la etapa, dotando al profesorado de competencias para la mejora o perfeccionamiento en el modelo de conducta y que se encuentre competente para dar respuesta a las necesidades formativas y afectivas que les demande el alumnado de esta etapa.

Nuestra investigación no pretende establecer leyes generales, sino ofrecer una aportación que pueda ser utilizada como punto de partida para futuras investigaciones que tengan como objetivo de estudio la validación de programas que trabajen el desarrollo de competencias emocionales y que permitan alcanzar un mejor bienestar personal y adaptación social, menores niveles de burnout y mayores de engagement en el profesorado de Educación Infantil. Concretamente, nuestros hallazgos pretenden poner de manifiesto la necesidad 
de fomentar las competencias emocionales de los docentes, sin menoscabo de otro tipo de intervención más organizacional, como forma de mejorar su calidad de vida laboral y el desempeño de su labor docente.

Entendemos que la principal peculiaridad que aporta originalidad y relevancia a nuestro trabajo es el de habernos centrado en el estudio de los niveles de burnout del profesorado de Educación Infantil dado que es una etapa poco explorada respecto al síndrome en nuestro país, siempre con la intención de detectar competencias que, tanto en su formación inicial como continua, necesitan ser mejoradas.

Por tanto, a la luz de los resultados obtenidos podemos interpretar que si se mejora la competencia percibida referida al grado en el que el sujeto cree poder regular sus estados emocionales negativos y prolongar los positivos (dimensión Reparación de $(\mathrm{E})$, esto produciría una reducción de los niveles de cansancio emocional en su trabajo (Agotamiento emocional) y mayores niveles sentimientos de competencia y eficacia en el trabajo (Realización personal).

Los resultados obtenidos sugieren que el profesorado de Educación Infantil de la Bahía de Cádiz muestra unos niveles reducidos de burnout. Estos, se caracterizan por la ausencia en la pérdida de recursos emocionales debido a las demandas del alumnado y la ausencia de sentimientos de estar saturado por el trabajo (Agotamiento emocional), también se caracterizan por percibirse con unos altos niveles de sentimientos de competencia y eficacia en el trabajo (Realización personal). Los sujetos de nuestra muestra no presentan tendencia a evaluar el propio trabajo de forma negativa, ni vivencian una insuficiencia profesional.

En este sentido y a la luz de los resultados obtenidos, en relación con el objetivo de nuestro estudio, se presentan las siguientes conclusiones ajustadas a las características concretas de nuestra muestra. En primer lugar, los docentes de Educación Infantil de la Bahía de Cádiz presentan índices reducidos de burnout en las dimensiones contempladas. Asimismo, se perciben con unos niveles altos de competencias emocionales en sus tres dimensiones. Por otro lado, la percepción de autoeficacia en la competencia emocional Reparación, actúan como factor de protección ante el síndrome de burnout. En consecuencia, en el diseño de programas formativos dirigidos al profesorado de Educación Infantil deberían incluirse contenidos enfocados al desarrollo de la Reparación (dimensión de IE) que actuarían como factor de protección ante el burnout por el efecto directo sobre sus dimensiones, concretamente sobre dos de sus tres dimensiones: el Agotamiento emocional y la Realización Personal. Finalmente, en aquellos colectivos en los que aparezcan síntomas del síndrome de burnout relacionado con unos niveles competenciales bajos en Inteligencia Emocional, podrían y deberían ser implementados programas de formación encaminados a la mejora de la IE tanto en el período de formación inicial como durante la formación permanente.

Como principales limitaciones del estudio se encuentran el tamaño de la muestra y su concreción en una zona geográfica delimitada como es la Bahía de Cádiz. Asimismo, contemplar las posibles variables que pueden estar implicadas en el desarrollo del síndrome burnout, tales como, cargas familiares, número de años desempeñando labores docentes, desempeño de roles de género en las labores domésticas e incluso la disponibilidad de ayuda externa para la realización de dichas labores, o sí desempeñan un cargo de gestión.

\section{Conflicto de intereses}

Los autores declaran no tener ningún conflicto de intereses.

\section{REFERENCIAS}

Alberici, A., y Serreri, P. (2005). Competencias y formación en la edad adulta. El Balance de competencias. Barcelona: Alertes.

Anderson, M. B. G. e Iwanicki, E. F. (1984). Teacher motivation and its relationship to burnout. Education Administration Quarterly, 20, 109-132. doi:10.1177/0013161X84020002007

Arís-Redó, N. (2009). El síndrome de burnout en los docentes. Electronic Journal of Research in Educational Psychology, 18, 829-848. 
Austin, E. J., Saklofske, D. H., y Mastoras, S. M. (2010). Emotional intelligence, coping and exam-related stress in Canadian undergraduate students. Australian Journal of Psychology, 62, 42-50. doi:10.1080/00049530903312899

Bandura, A. (1997). Self-efficacy: The exercise of control. New York: Freeman.

Bandura, A. (1999). Ejercicio de la eficacia personal y colectiva en sociedades cambiantes. En A. Bandura (Ed.), Autoeficacia: Cómo afrontamos los cambios de la sociedad actual (pp. 19-54). España: Desclée de Brouwer.

Bandura, A. (2004). Health promotion by social cognitive means. Health Education \& Behavior, 37(2), 143-164. doi: $10.1177 / 1090198104263660$

Bermúdez, R., Martínez, G., Ríus, F., y Esteve, J. M. (2004). Relaciones entre el uso de la voz y el burnout en los docentes de Educación Infantil y Primaria de Málaga. Revista Española de Pedagogía, 227, 85-102.

Boyatzis, R., McKee, A., y Goleman, D. (2002). Reawakening your passion for work. Harvard Business Review. 80(4). 86-94.

Brackett, M. A., Mayer, J. D., y Warner, R. (2004). Emotional Intelligence and its relation every day. Personality and individual Differences, 36, 1387-1402. doi:10.1016/ S0191-8869(03)00236-8

Brackett, M. A., y Katulak, N. A. (2006). Emotional Intelligence in the classroom: Skill-based training for teacher and students. En J. Ciarrochi y J. D. Mayer (Eds.), Improving emotional intelligence: a practitioners guide (pp. 1-27). New York: Psychology Press/ Taylor \& Francis.

Capelo, R, Pocinho, M., y Santos, C. (2013). Stresse e Coping em educadoras de infância portuguesas: um estudo exploratório. Trabajo, 28, 69 - 81. doi:10.1590/01044060.37870

Capelo, R. y Pocinho, M. (2014). Autoeficácia docente: predição da satisfação dos professores. Educar em Revista, 54, 175184.

Christopher, G., y Thomas, M. (2009). Social problem solving in chronic fatigue syndrome: Preliminary findings. Stress and Health, 25(2), $161-169$.

Cordeiro, J. A., Guillén, C. L., Gala, F. J., Lupiani, M., Benítez, A., y Gómez, A. (2003). Prevalencia del Síndrome de burnout en los maestros. Resultados de una investigación preliminar. Recuperado 9 Febrero, 2016, de http://www.psiquiatria.com/revistas/index. $\mathrm{php/psicologiacom/article/view/794/}$

Donaldson-Feilder, E. J., y Bond, F. W. (2004). The relative importance of psychological acceptance and emotional intelligence to workplace well-being. British Journal of Guidance and Counselling, 33(3), 187-203. Durán, A., Extremera, N. y Rey, L. (2004): Engagement and Burnout: Analysing their association patterns. Psychological Reports, 94, 1048-1050.

Duran, M. A., Extremera, N., Montalban, F. M., y Rey, L. (2005). Engagement y Burnout en el ámbito docente: Análisis de sus relaciones con la satisfacción laboral y vital en una muestra de profesores. Revista de Psicología del Trabajo y de las Organizaciones, 21 (12), 145-158.

Extremera, N., Fernández-Berrocal, P., y Durán, M. A. (2003). Inteligencia emocional y burnout en profesores. Encuentros en Psicología Social, 1, 260-265.

Extremera, N., Rey, L., y Pena, M. (2010) La docencia perjudica seriamente la salud. Análisis de los síntomas asociados al estrés docente. Boletín de Psicología, 100, 43-54.

FETE-UGT. (2004). Catálogo de enfermedades profesionales de los docentes. Madrid: Observatorio permanente de riesgos psicosociales de UGT.

FETE-UGT. (2006). Guía de prevención de riesgos psicosociales en el sector de la enseñanza. Recuperado el 15 de febrero de 2016 de http://portal.ugt.org/saludlaboral/ observatorio/publicaciones/cuader guias/2006 Guia_sectorial Ensenanza. pdfMadrid.

FETE-UGT. (2014) 28 de Abril, Día Internacional de la Salud y Seguridad en el trabajo [Comunicado de prensa]. Recuperado el 15 
de febrero de 2016 de http://www.feteugtcyl. es/sites/default/files/COM28\%20abril FETE.pdf

Fernández-Berrocal, P., Extremera, N., y Ramos, N. (2004). Validity and reliability of the Spanish modified version of the Trait Meta-mood Scale. Psychological Reports, 94, 751-755.

Giardini, A., y Frese, M. (2006). Reducing the negative effects of emotion work in service occupations: Emotional competence as a psychological resource. Journal of Occupational Health Psychology, $17(1), 63$ 75.

Gil-Monte, P. R. (2001). El síndrome de quemarse por el trabajo (síndrome Burnout): aproximaciones teóricas para su explicación y recomendaciones para la intervención. Recuperado el 15 de febrero de 2016, de http://psicopediahoy.com/burnout-teoriaintervencion/

Goleman, D. (1995). Inteligencia Emocional. Barcelona: Kairós.

Guerrero, E., y Rubio, J. C. (2005). Estrategias de prevención e intervención del Burnout en el ámbito educativo. Salud Mental, 28(5), 27-33.

Hughes, J. (2005). Bringing emotion to work: Emotional Intelligence, employee resistance and the reinvention of character. Work Employment and Society, 19(3), 603-626.

Limonero, J., Tomás-Sábado, J., FernándezCastro, J., y Gómez-Benito, J. (2004). Influencia de la inteligencia emocional percibida en el estrés laboral de enfermería. Ansiedad y estrés, 10, 29-41.

Maslach, C., y Jackson, S. E. (1986). The Maslach Burnout Inventory. Zaed, Palo Alto: Consulting Psychologists Press.

Maslach, C., y Jackson, S. E. (1981). The measurement of experienced burnout. Journal of Occupational Behavior, 2(99), 99-113.

Matud, M. P., García, M. A., y Matud, M.J. (2002). Estrés laboral y salud en el profesorado: un análisis diferencial en función del género y del tipo de enseñanza. Internation Journal of clinical and Health
Psychology, 2, 251-465.

Mikolajczak, M., Nelis, D., Hansenne, M., y Quoidbach, J. (2008). If you can regulate sadness, you can probably regulate shame: Associations between trait emotional intelligence, emotion regulation and coping efficiency across discrete emotions. Personality and Individual Differences, 44(6), 1356-1368.

Navío, A. (2005). Propuestas conceptuales en torno a la competencia profesional. Revista de Educación, 337, 213-234.

Nikolaou, I., y Tsaousis, I. (2002). Emotional Intelligence in the Workplace: Exploring its effects on Occupational Stress and Organizational Commitment. International Journal of Organizational Analysis, 10, 327 342.

Nunes, M., y Farias, O. (2008). Funcionamento e desenvolvimento das crenças de autoeficácia: uma revisão. Revista Brasileira de Orientação Profissional, 9(1), 29-42.

Orden ECl/3854/2007, de 27 de diciembre, por la que se establecen los requisitos para la verificación de los títulos universitarios oficiales que habiliten para el ejercicio de la profesión de Maestro en Educación Infantil.

Pena, M., y Extremera, N. (2012). Inteligencia emocional percibida en profesorado de primaria y su relación con los niveles de burnoute ilusión porel trabajo (engagement). Revista de Educación, 359, 604-627.

Pocinho, M., y Capelo, R. (2009). Vulnerabilidade ao stress, estratégias de coping e autoeficácia em professores portugueses. Educacao e Pesquisa, 35(2), $351-367$.

Pocinho, M., y Peresterelo, M. C. (2011). Um ensaio sobre Burnout, Engagement e estratégias de Coping na Profissão Docente. Educação e Pesquisa, 37(3), 513-528. doi: 10.1590/S1517-97022011000300005

Salanova, M., y Grau, R. (1999). Burnout and new technologies at work. Simposium "New Directions in Burnout Research". The $9^{\text {th }}$ West European Congress on Work and Organizational Psychology. Espoo-Helsinki, Mayo 1999. 
Salovey, P., Mayer, J. D., Goldman, S., Turvey, C., y Palfai, T. (1995). Emotional attention, clarity, and repair: Exploring emotional intelligence using the Trait Meta-Mood Scale. En J. W. Pennebaker (Ed.), Emotion, disclosure, and health (pp. 125-154). Washington, DC: American Psychological Association.

Schwab, R. L., Jackson, S. E., y Schuler, R. S. (1986). Educator Burnout: Sources and Consequences. Educational Research Quarterly, 10(3), 14-30.
Soares, G. y Pocinho, M. (2013). Emoções e liderança escolar: um estudo com conselhos executivos das escolas públicas portuguesas. Revista Iberoamericana de Educacion, 61 (4), 1-15.

Vanderbenghe, R. y Huberman, A. M. (1999). Understanding and preventing teacher burnout: a sourcebook of international research and practice. Cambridge: University Press 